\title{
Lamotrigine Induced Dystonia in a Patient with Bipolar Affective Disorder Ratan J Lihite ${ }^{1 *}$, Mangala Lahkar ${ }^{1}$, Deepanjali Medhi² and Rahul Mathur ${ }^{2}$
}

${ }^{1} A D R$ Monitoring Centre, Department of Pharmacology, Gauhati Medical College, Guwahati-32, India

${ }^{2}$ Department of Psychiatry, Gauhati Medical College, Guwahati-32, India

\section{Dear Sir,}

Lamotrigine is a first drug since lithium approved for maintenance treatment of bipolar type I. Lamotrigine-induced extrapyramidal side effects are rarely reported in literatures. In this letter, we have presented a case of Bipolar disorder which has induced dystonia to lamotrigine. Lamotrigine is a phenyltriazine derivative [1] which was originally approved by USA Food \& Drug Administration for partial complex and generalized seizures. It was also recommended for treating bipolar affective disorder by British association for psychopharmacology in 2009 [2]. Lamotrigine is generally well tolerated. However, the most common adverse effects due to lamotrigine are nausea, headache, dizziness, ataxia, diplopia, somnolence, and tremor. Beside these, skin eruptions, arthralgia, haematological abnormalities comprised of leucopenia and thrombocytopenia, and elevated liver enzymes are also reported [3]. Some psychiatric problems are also associated with lamotrigine use, which are rarely reported in literature.

In this letter, a male patient of 21 year was reported from psychiatry department of Gauhati medical college and hospital, Guwahati, Northeast India. There was no history of psychotic symptoms and family history of mental illness. He had history of sleep decreased, nocturnal awakenings, loss of appetite and weight, hearing voices, and low mood. He was born by natural vaginal delivery, with no perinatal complications. He was well-kempt and cooperative. His speech was also spontaneous and fluent. He had reactive affect and negative cognition during periods of low mood. He was also expressing death wish. Physical examination was always normal, but with noticeable weight loss during his certain low mood exacerbations. He was admitted to this hospital in psychiatry ward which was referred for psychiatry evaluation with anxiety and depressive symptoms and failure to respond antidepressant treatment which was prescribed by general practitioner. He was diagnosed with bipolar affective disorder and failure to achieve remission of symptoms on two different antipsychotic medications (quetiapine and olanzapine) and anticonvulsant medication (valproic acid).

It was decided to introduce lithium carbonate with gradual titration and he perceived improvement in his symptoms and mood stabilised. However, few months later he reported relapse in his symptoms despite adequate lithium level. Lamotrigine was introduced as add-on therapy to lithium with $25 \mathrm{mg}$ daily for two weeks and later on it was increased to $50 \mathrm{mg}$ /day. A few days after lamotrigine dose was increased to 100 $\mathrm{mg}$ /day, thereafter patient showed agitated behaviour, and experienced disturbed sleep. Patient also had pain and stiffness in his neck and jaw along with muscle spasms in extremities. Upon learning these symptoms, the dose of lamotrigine was reduced to $50 \mathrm{mg}$ which resulted into immediate reduction in the intensity of neck and jaw dystonic reactions. After few days, lamotrigine with $100 \mathrm{mg}$ daily dose was again reintroduced with an aim to stabilize his mood and next day he again developed acute dystonic reactions. Thus, these reoccurrence of symptoms were suspected to cause by lamotrigine, and were eventually subsided after 1 week with lamotrigine withdrawal. Response to withdrawal of drug was clinically reasonable and phenomenologically found definite. Event was with plausible time relationship to drug intake and was unlikely to be attributed to disease. Therefore, by WHOUppsala Monitoring Centre (UMC) causality assessment system [4], the causal relationship between these reaction and lamotrigine was certain whereas by Naranjo probability assessment algorithm [4], it was found probable.

Antipsychotic agent-induced extrapyramidal side effects are well known and are most commonly reported adverse effect during clinical practice. However, anticonvulsant drugs induced extrapyramidal symptoms are rarely reported due to lack of awareness about this drug reaction [5]. In this letter, patient has induced dystonia comprised of stiffness and muscle spasms over neck and jaw involving extremities. These symptoms are usually induced by antipsychotic drugs that antagonize dopamine $\mathrm{D}_{2}$ receptors. Moreover, co-administration of lithium and lamotrigine at higher doses hasn't precipitated extrapyramidal symptoms in majority of the bipolar patients. However, this patient has developed acute dystonia after dose increment of lamotrigine in the presence of lithium. Even if the prodystonic effect of lithium acted synergistically to develop dystonic reaction, it seems that inclusion of lamotrigine in the treatment was the catalyst. Thus, this increase in dosage may likely to have aggravated his symptoms, which were subsided soon after discontinuing lamotrigine. Lamotrigine induced dystonia was initially reported in hamster model by Richter et al. [6]. It was reported that inhibition of GABA release and lack of selectivity for glutamate release inhibition are critically involved in prodystonic tendencies of lamotrigine. This excessive glutamate release may over stimulate the brain and introduce excitotoxicity [6]. In a review of literature of Villari et al. case reports of psychiatric symptoms related to lamotrigine use are presented in four categories; affective switches in depressive patients, hallucinations in depressive patients, psychotic episodes in patients with epilepsy and hallucinations in a patient with epilepsy [7]. Most of these cases were concluded on the basis of favourable course showed by the patient following withdrawal of lamotrigine. Similarly in this patient, dystonia was subsided upon withdrawal of lamotrigine. However, further studies are required which can provide underlying mechanism in lamotrigine-induced dystonia.

\section{References}

1. Hameed $Y$, Hamelijnck $J(2014)$ Lamotrigine-induced hallucination in patient with bipolar disorder and no history of epilepsy or psychosis: a case report and literature review. $\mathrm{Br} \mathrm{J}$ Med Pract 7: a714.

2. Goodwin GM (2009) Evidence-based guidelines for treating bipolar disorder: revised second edition-recommendation from the British Association for Psychopharmacology. J Pychopharmacol 23: 346-388.

*Corresponding author: Ratan J Lihite, ADR Monitoring Centre (NCC-PvPI, Indian Pharmacopoeia Commission, Ministry of Health and Family welfare, Govt. of India), Department of Pharmacology, Gauhati Medical College, Indrapur, Guwahati-32, India, Tel: +91 9706143510; E-mail: r.lihite@yahoo.com

Received March 07, 2016; Accepted April 13, 2016; Published April 16, 2016

Citation: Lihite RJ, Lahkar M, Medhi D, Mathur R (2016) Lamotrigine Induced Dystonia in a Patient with Bipolar Affective Disorder. Brain Disord Ther 5: 212. doi:10.4172/2168-975X.1000212

Copyright: @ 2016 Lihite RJ, et al. This is an open-access article distributed under the terms of the Creative Commons Attribution License, which permits unrestricted use, distribution, and reproduction in any medium, provided the original author and source are credited. 
Citation: Lihite RJ, Lahkar M, Medhi D, Mathur R (2016) Lamotrigine Induced Dystonia in a Patient with Bipolar Affective Disorder. Brain Disord Ther 5: 212. doi:10.4172/2168-975X.1000212

Page 2 of 2

3. http://www.mims.com/INDIA/drug/info/lamotrigine

4. Belhekar MN, Taur SR, Munshi RP (2014) A study of agreement between the Naranjo algorithm and WHO-UMC criteria for causality assessment of adverse drug reactions. Indian J Pharmacol 46:117-120.

5. Marrero-Gonzalez PC, Ruano OL, Catalano G, Catalano MC (2014) Dystonia associated with lamotrigine therapy: a case report and review of the literature.
Curr Drug Saf 9: 60-62.

6. Richter A, Loschmann PA, Loscher W (1994) The novel antiepileptic drug lamotrigine, exerts prodystonic effects in a mutant hamster model of generalized dystonia. Eur J Pharmacol 264: 345-351.

7. Villari V, Rocca P, Frieri T, Bogetto F (2008) Psychiatric symptoms related to the use of lamotrigine: a review of the literature. Funct Neurol 23: 133-136. 